\title{
MONOFUNCIONALIDAD, MULTIFUNCIONALIDAD E HIBRIDACIÓN DE FUNCIONES DE LAS AGRICULTURAS EN LA CUENCA DEL RÍO \\ GUAGUARCO, SUR DEL TOLIMA
}

Álvaro Acevedo-Osorio ${ }^{1}$

Recibido el 18 de junio de 2015, aprobado el 30 de octubre de 2015 y actualizado el 19 de mayo de 2016

DOI: 10.17151/luaz.2016.43.12

\section{RESUMEN}

El rol monofuncional de la agricultura empresarial ha generado serios desequilibrios ambientales y socioculturales que comprometen su productividad futura. Una valoración de la multifuncionalidad de la agricultura en 18 sistemas tradicionales, ganaderos y de monocultivos, evaluados en la cuenca del río Guaguarco a partir de variables biofísicas, sociales, culturales, productivas y financieras, calificaron la agricultura tradicional como la más funcional, seguida de los sistemas ganaderos y de monocultivo. La capacidad de los agricultores para desplegar múltiples funciones en sus sistemas de finca constituye una estrategia para enfrentar las adversidades que ponen en riesgo su continuidad.

\section{PALABRAS CLAVE}

Multifuncionalidad, agricultura tradicional, sistemas de finca, funciones múltiples de la agricultura. 


\title{
MONOFUNCIONALITY, MULTIFUNCIONALITY AND HYBRIDIZATION OF AGRICULTURE'S FUNTIONS OF THE GUAGUARCO RIVER BASIN IN THE SOUTHERN TOLIMA
}

\begin{abstract}
The monofunctional role of the business-oriented agriculture has generated serious environmental and socio-cultural imbalances that threaten its future productivity. An appraisal of agriculture's multifunctionality in 18 traditional farming systems, livestock farming and monoculture farming, evaluated at the Guaguarco river basin with biophysical, social, cultural, productive and financial variables, evaluated the traditional farming systems as the most functional followed by the livestock farming and monoculture farming. The farmer's capability to deploy multiple functions to their farming systems, constitute a strategy to face the adversities that put at risk continuity.
\end{abstract}

\section{KEY WORDS}

Multifunctionality, traditional farming, farming systems, multiple functions of the agriculture.

\section{INTRODUCCIÓN}

Los conceptos dominantes del desarrollo rural no pueden explicar la complejidad de los retos actuales relacionados con la agricultura y el mundo rural (Samper \& Torrens, 2015). La visión productivista, que la sociedad y el mercado han dado históricamente a la agricultura y al desarrollo rural, está teniendo un cambio importante en la actualidad. Los problemas estructurales del sector rural como pobreza, exclusión, riesgos asociados al cambio climático, degradación ambiental, crisis energética y tecnológica, entre otros, tienen causas complejas que requieren de creatividad, nuevas competencias sociales y destrezas específicas para enfocar la dinámica hacia el cambio que caracteriza el desarrollo multidimensional (Steiner \& Posch, 2006). 
La apreciación reduccionista de la agricultura, centrada en lo productivo, desconoce el rol multifuncional que históricamente ha cumplido (Holmes, 2006; Moyano, 2008), ya que aparte de contribuir con la economía, se ocupa de la protección del hábitat y la biodiversidad, la conservación del patrimonio cultural, el aseguramiento de condiciones de vida digna para las familias y comunidades rurales, la generación de oportunidades para la recreación y el ocio creativo, entre otras.

Más allá de resultados económicos, la agricultura genera impactos diversos sobre el medio ambiente y la sociedad no visibilizados ni cuantificados. Estos efectos van desde los que ocasionan un impacto fuertemente negativo sobre los recursos naturales, las comunidades y la sociedad, hasta los que favorecen condiciones para la generación de mayor bienestar.

Son escasos los esfuerzos dedicados a analizar, de manera holística, los efectos de la agricultura y la manera como los agricultores estructuran y operan diversas formas de agricultura en un territorio (Ploeg, 2008). En general, recurren a diversas estrategias y asignan a sus sistemas de finca un amplio rango de funciones, que les permite encontrar soluciones a los múltiples problemas que enfrentan (Toledo, 1993; Bjørkhaug \& Richards, 2008; Baudel, 2009).

La multifuncionalidad constituye un atributo de la agricultura y los territorios (Moyano, 2008) en tanto generan no solo productos mercadeables sino también bienes y servicios no ponderados monetariamente. La multifuncionalidad permite reconocer y valorar los múltiples aportes de la agricultura, facilitando la sustentabilidad social y ambiental tanto de la agricultura como de los territorios (Gerritsen, Rosales, Moreno \& Martínez, 2006; Bjørkhaug \& Richards, 2008). Propuesta en la Agenda 21 (Cumbre de la Tierra de Río de 1992), la multifuncionalidad ha ganado una importancia creciente en el campo científico y político sobre el futuro de la agricultura.

Algunos autores (Renting et al., 2009) argumentan que la multifuncionalidad debe convertirse en un nuevo metanivel para el análisis y la toma de decisiones relacionadas a la agricultura y los territorios, otros opinan que este concepto 
requiere ser desarrollado y aplicado al nivel de finca, como la más pequeña unidad espacial de análisis desde la cual se configura el territorio (Wilson, 2009).

En Europa el concepto está ligado al ámbito de decisiones políticas respecto a los subsidios para el desarrollo agrícola (OECD, 2001) y más recientemente a la manera de estructurar nuevas empresas que no solo se fijen metas productivas sino que valoren también la prestación de servicios ecológicos (Jordan \& Warner, 2010), mientras que en América Latina resulta un concepto poco conocido, con símiles en postulados como las "estrategias de uso múltiple" (Toledo, AlarcónCháires \& Barón, 2009) que emplean los agricultores para apropiarse de los paisajes y sus recursos bióticos y físicos para satisfacer sus requerimientos básicos.

Un enfoque sobre la multifuncionalidad más relacionado a las condiciones de la agricultura en el neotrópico, ha sido formulado por Rivas \& Quintero (2014) como Funciones Múltiples de la Agricultura (FMA) para reconocer las formas propias de operar que tienen los agricultores tradicionales sobre sus sistemas de finca reconociendo sus estrategias adaptativas.

En la racionalidad campesina, la agricultura representa un fenómeno multifacético de apropiación de la naturaleza que permite a los agricultores la producción de una enorme cantidad de productos de uso propio y para el intercambio económico con el mercado (Toledoet al., 2009). Con los recursos obtenidos en el mercado, por la venta o trueque de sus productos, los agricultores obtienen bienes manufacturados (Toledo, 1993; Toledo \& Barrera-Bassols, 2008) para cubrir otras necesidades de la familia, no solventadas por la agricultura. Esta racionalidad promueve en sí la multifuncionalidad del espacio rural.

La aplicación del enfoque de la multifuncionalidad implica diseñar y validar sistemas de finca innovadores que promuevan diversas funciones; obliga también a repensar la generación de nuevas formas del conocimiento (Bjørkhaug \& Richards, 2008; Labarthe, 2009; Jordan \& Warner, 2010). Los retos de investigación sugeridos van más allá de los alcances de enfoques disciplinares 
simples que no pueden incorporar plenamente todas las diferentes perspectivas requeridas para analizar la multifuncionalidad (Renting et al., 2009).

Dado que los actuales programas de desarrollo rural en Colombia son estructurados desde una visión exclusivamente productivista (Perry, Barberi \& Garay, 2013) y, por lo tanto monofuncional, se hace necesario abordar análisis multidimensionales que analicen las diversas funciones de la agricultura en un territorio y las integren en la planificación de políticas públicas y programas que incluyan aspectos productivos, ambientales y socioculturales, además de los financieros.

Esta investigación realizó un análisis comparativo de las funciones financieras, productivas, biofísicas, culturales y sociales de tres formas de agricultura presentes en la cuenca del río Guaguarco (sur del Tolima, Colombia), destacando los diversos grados de funcionalidad y la racionalidad implícita en las formas de manejo implementadas por los agricultores.

\section{METODOLOGÍA}

El estudio se realizó en la cuenca del río Guaguarco, localizada entre los municipios de Coyaima y Natagaima al sur del departamento del Tolima sobre el flanco derecho de la cordillera Central Colombiana. Fueron seleccionados 18 "sistemas de finca" $=$ representativos de cada uno de las tres principales formas de agricultura en la cuenca: 6 unidades indígenas (UI), 6 unidades familiares (UF) y 6 unidades empresariales (UE), seleccionados de acuerdo a la forma de tenencia de la tierra, estructura del sistema (cultivo predominante), operación del mismo (tipo de mano de obra empleada) y destino de la producción (Figura 1). 


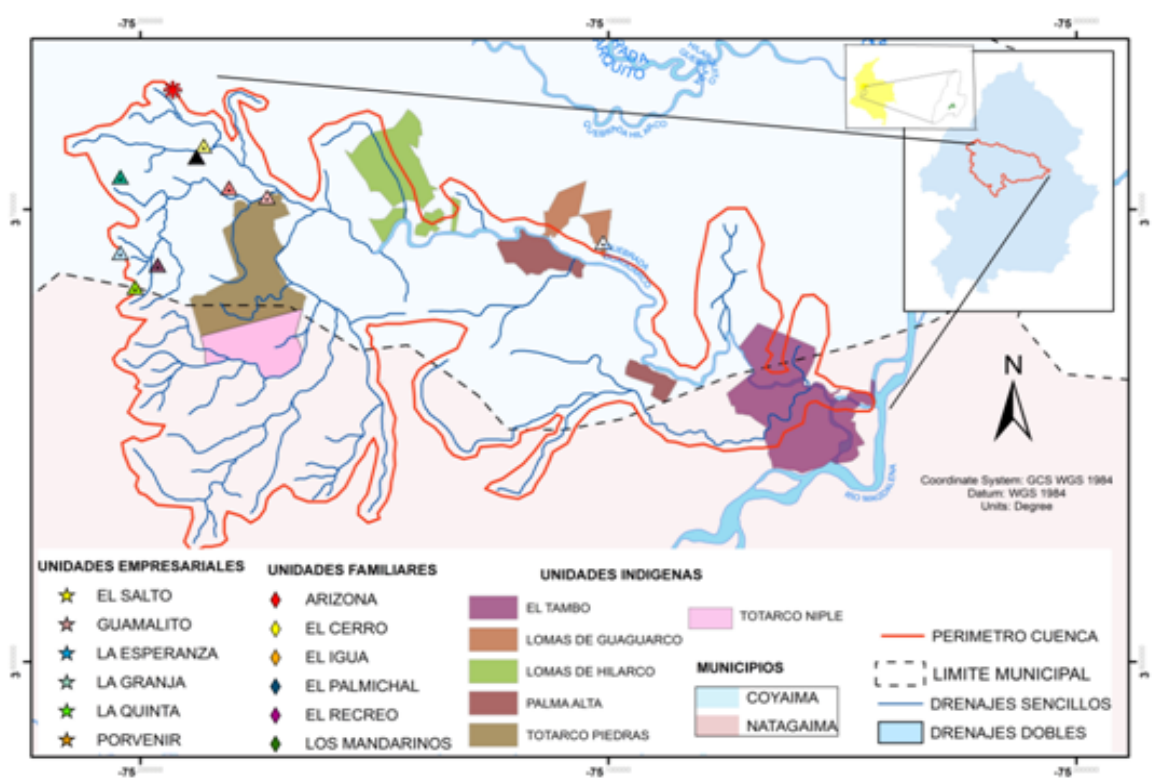

Fuente: editado por Camilo Alvarez, a partir de digitalización sobre mapa físico del Distrito de Riego El Triángulo del Tolima (SNC Lavalin International), información cartográfica del IGAC y SIGOT/IGAC.

Figura 1. Ubicación de las unidades de finca estudiadas en la cuenca del río Guaguarco, sur del Tolima.

Se desarrolló como investigación cualitativa de carácter interpretativo (Gómez, Deslauriers \& Alzate, 2010) empleando el método de estudio de caso, buscando la comprensión detallada de la realidad construida por los agricultores en su entorno.

La investigación se desarrolló en dos fases: 1) caracterización de los sistemas de finca y 2) evaluación de la multifuncionalidad de la agricultura. Para la primera fase se tomaron 22 características representativas de los cinco subsistemas sugeridos por Fleskens, Duarte \& Eicher (2009) y Gómez-Sal \& González (2007): biofísico, productivo, financiero, social y cultural (Tabla 1). 
Tabla 1. Características consideradas para la conformación de conglomerados

\begin{tabular}{|c|c|c|c|c|}
\hline $\begin{array}{l}\text { Subsistema } \\
\text { productivo }\end{array}$ & $\begin{array}{l}\text { Subsistema } \\
\text { financiero }\end{array}$ & Subsistema social & Subsistema cultural & $\begin{array}{l}\text { Subsistema } \\
\text { biofisico }\end{array}$ \\
\hline $\begin{array}{l}\% \text { del área en } \\
\text { monocultivo } \\
\text { comercial }\end{array}$ & $\begin{array}{l}\text { Excedente familiar } \\
\text { integral por familia } \\
\text { por mes ( } \$ \text { ) }\end{array}$ & $\begin{array}{l}\text { Tipo de tenencia } \\
\text { de la tierra }\end{array}$ & $\begin{array}{l}\text { Relación cultural con } \\
\text { la tierra }\end{array}$ & $\begin{array}{l}\text { \% área en } \\
\text { conservación }\end{array}$ \\
\hline $\begin{array}{l}\text { \% del área en } \\
\text { plátano cachaco }\end{array}$ & $\begin{array}{l}\text { Relación } \\
\text { beneficio/costo } \\
\text { familiar }\end{array}$ & $\begin{array}{l}\text { Disponibilidad de } \\
\text { vivienda en el } \\
\text { predio }\end{array}$ & $\begin{array}{l}\text { Tipo de conocimiento } \\
\text { empleado en: }\end{array}$ & $\begin{array}{l}\% \text { materia } \\
\text { orgánica de los } \\
\text { suelos }\end{array}$ \\
\hline $\begin{array}{l}\% \text { del área en } \\
\text { policultivo } \\
\text { tradicional }\end{array}$ & & $\begin{array}{l}\% \text { de adultos } \\
\text { dedicados a la } \\
\text { producción en la } \\
\text { finca }\end{array}$ & $\begin{array}{l}\text { - Manejo de } \\
\text { - Fuelos } \\
\text { - Origen de la }\end{array}$ & $\begin{array}{l}\text { Indice estructural } \\
\text { del suelo }\end{array}$ \\
\hline $\begin{array}{l}\% \text { del área en } \\
\text { potrero }\end{array}$ & & $\begin{array}{l}\text { Tipo de mano de } \\
\text { obra empleada }\end{array}$ & $\begin{array}{l}\text { semilla } \\
\text { - Manejo de } \\
\text { arvenses } \\
\text { - Manejo de }\end{array}$ & $\begin{array}{l}\text { Ton/ha de } \\
\text { carbono } \\
\text { contenidas en el } \\
\text { suelo }\end{array}$ \\
\hline $\begin{array}{l}\mathrm{N}^{2} \text { de alimentos } \\
\text { producidos y } \\
\text { consumidos } \\
\text { frecuentemente por } \\
\text { la familia } \\
N^{2} \text { de actividades } \\
\text { productivas en la } \\
\text { finca }\end{array}$ & & & $\begin{array}{l}\text { plagas y } \\
\text { enfermedades }\end{array}$ & \\
\hline
\end{tabular}

Fuente: el autor.

El subsistema biofísico comprende los factores del ecosistema que determinan su calidad y su capacidad de seguir proveyendo los servicios de los cuales dependen la agricultura y la sociedad. Las características estudiadas en esta dimensión fueron: área destinada a conservación, principales especies de biodiversidad y agrobiodiversidad, contenidos de carbono orgánico y materia orgánica de los suelos e índice estructural del suelo.

Para el análisis de la conservación del suelo, la biodiversidad y la agrobiodiversidad, se analizan las áreas de conservación en cada finca que cubren y protegen el suelo y albergan la biodiversidad propia de la región así como las áreas destinadas a sistemas de policultivos tradicionales que también cumplen la función de proteger el suelo y albergar la agrobiodiversidad vinculada a los sistemas locales de producción de alimentos. Para estimar el porcentaje de Carbono Orgánico del Suelo en porcentaje (COS), se tomaron muestras de suelos de cada sistema de finca, 3 sub-muestras por finca, para homogenizar y obtener 
una muestra por cada sistema de finca que fue sometida a prueba colorimétrica de Walkley-Black en laboratorio de suelos. El valor de Materia Orgánica se obtuvo corrigiendo el \% COS por el factor de van Bemmelen que equivale a 1.724 (Hernández et al., 2011).

El índice estructural del suelo (Si) propuesto por Pieri (1995), mide el nivel de deterioro ocasionado por su grado de compactación o sellado del suelo y erosión hídrica. Se basa en el contenido de Materia Orgánica (MO) y las cantidades de limos y arcillas presentes y se midió de la siguiente manera: $\mathrm{Si}=\mathrm{MO}(\%) /($ arcilla + limo) $\% \times 100$. Los contenidos porcentuales de arcillas, limos y arenas se determinaron por el método de Bouyoucos. El índice discrimina entre suelos degradados ( $\mathrm{Si}<5$ ), suelos con alto riesgo de deterioro (Si entre 5-7), suelos con moderado riesgo (Si entre 7-9) y suelos estructuralmente estables (cuando $\mathrm{Si}>9$ ).

Se analizó el nivel de captura de carbono en el suelo, por ser esta una de las principales variables de los servicios ambientales que proveen los sistemas de finca frente a los riesgos asociados con el calentamiento climático, que fue calculado con la siguiente fórmula (Hernández et al., 2011):

$$
C(t / h a)=\% \operatorname{COS}^{*} \text { área }(m 2)^{*} \text { profundidad }(m)^{*} D \cdot A(t / m 3) / 100 .
$$

El área de referencia es el equivalente a una hectárea $\left(10.000 \mathrm{~m}^{2}\right)$; la profundidad se refiere a la capa arable que se toma equivalente a 0,20 m. D.A = Densidad Aparente, se estimó considerando los porcentajes de las partículas arcillas, limos y arenas.

El subsistema productivo está relacionado a la producción usada para el autoconsumo familiar, el intercambio o trueque y el mercado. Incluyó las características: grado de abastecimiento, producción para el mercado, producción de insumos o recursos propios para la producción, otras actividades productivas como artesanías, recursos madereros, alimentos procesados, leña, etc. 
El subsistema financiero entendido como las maneras empleadas por las familias agricultoras para generar ingresos económicos. Incluyó las características: costos e ingresos, conformación del ingreso familiar y comunitario (Forero, 2002) considerando producción de alimentos, materias primas o ingresos no agropecuarios, excedentes económicos por cada sistema agropecuario. Como característica de rentabilidad, se determina la relación beneficio/costo obtenida en la división entre los Ingresos Agropecuarios Brutos y los costos totales (domésticos y monetarios).

El subsistema social comprende todas aquellas acciones tendientes a generar bienestar para las familias y comunidad con enfoque de equidad intergeneracional y de género. Incluyó las características: composición familiar, forma de tenencia de la tierra, relaciones de género, tipo de mano de obra empleada en los sistemas de finca, vinculación de la familia, condiciones de habitabilidad, disponibilidad de servicios y tipo de construcción en la vivienda.

El subsistema cultural conformado por el conjunto de representaciones sociales o formas comunes de actuar que identifica a un grupo social, los juicios y creencias que se expresan en comportamientos interiorizados (Aguirre, 1998) o los elementos simbólicos que hacen parte de su identidad y que determinan su forma particular de relacionamiento, aprovechamiento, cuidado o protección de la naturaleza; lo que a su vez recrea y fortalece la identidad cultural del grupo social. Incluyó las características: criterios de innovación, hibridación o tradición en la implementación de técnicas de manejo agrícola, representaciones sociales vinculadas a los sistemas de finca. Para el caso de este estudio se acogieron las distinciones entre innovación (técnicas derivadas del proceso de tecnificación impulsado por profesionales e instituciones agrarias), tradición (prácticas ancestrales transmitidas entre los agricultores) e hibridación (combinación de técnicas con tradiciones) (Murillo, 2010), para analizar la manera se configuran las operaciones productivas a nivel de cada sistema de finca tomando como referencia 5 actividades productivas: manejo de suelos, fertilización, tipo de semilla empleada, manejo de arvenses y manejo de plagas y enfermedades.

Los datos de esta caracterización fueron sometidos a análisis estadístico no paramétrico tipo "clúster" o de "conglomerados" para establecer grupos con 
tendencias similares en el comportamiento de las diversas variables. Este análisis se realizó empleando el programa SPSS (Statistical Package of Social Sciences) versión 20.

La metodología y las variables seleccionadas para la segunda fase se basaron en las propuestas metodológicas de Gómez-Sal \& González (2007), Fleskens et al. (2009), Lovell et al. (2010), Murillo (2010), Licona (2012) y Andersen, Vejre, Dalgaard \& Brandt (2013) (Tabla 2) que seleccionan variables por dimensiones, o grupos de funciones, determinan rangos o atributos para cada variable y agregan los valores parciales obtenidos de cada una.

Las variables fueron cuantitativas y cualitativas; para poder agregar valores se llevaron a una misma escala de medición propuesta por Lovell et al. (2010) asignando una valoración entre +2 y -2 para determinar si la contribución de la variable es fuertemente positiva $(+2)$, levemente positiva $(+1)$, neutra $(0)$ levemente negativa $(-1)$ o fuertemente negativa $(-2)$. 
Tabla 2. Variables para evaluar las funciones múltiples de la agricultura de los sistemas de finca

\begin{tabular}{|c|c|c|c|c|}
\hline Subsistema & Variables & Concepto de la variable & $\begin{array}{ll}\text { Tipo de } & \text { de } \\
\text { variable } & \end{array}$ & $\begin{array}{l}\text { Unidad de } \\
\text { medida }\end{array}$ \\
\hline \multirow[b]{2}{*}{ Biofísico } & $\begin{array}{l}\text { Contenidos de } \\
\text { materia } \\
\text { orgánica }\end{array}$ & $\begin{array}{l}\text { Cantidad de materia orgánica } \\
\text { contenida en los suelos del } \\
\text { sistema de finca }\end{array}$ & Cuantitativo & $\%$ \\
\hline & $\begin{array}{l}\text { Cobertura de } \\
\text { conservación }\end{array}$ & $\begin{array}{l}\text { Porcentaje del área total con } \\
\text { coberturas de conservación: } \\
\text { cultivos multiestratificados y } \\
\text { zonas con vegetación silvestre } \\
\text { que cubran el suelo y tiendan a } \\
\text { promover la mayor biodiversidad }\end{array}$ & Cuantitativo & $\%$ \\
\hline \multirow{2}{*}{ Productivo } & $\begin{array}{l}\text { Diversificación } \\
\text { productiva }\end{array}$ & $\begin{array}{l}\text { Generación de productos y } \\
\text { servicios para el } \\
\text { autoabastecimiento yeneración } \\
\text { de recursos económicos }\end{array}$ & Cuantitativo & $N^{2}$ \\
\hline & $\begin{array}{l}\text { Abastecimiento } \\
\text { alimentario }\end{array}$ & $\begin{array}{l}\text { Manera como la familia se } \\
\text { asegura los alimentos necesarios } \\
\text { para su bienestar }\end{array}$ & Cuantitativo & $N^{2}$ \\
\hline Financiero & $\begin{array}{l}\text { Excedente } \\
\text { familiar integral } \\
\text { mensual }\end{array}$ & $\begin{array}{l}\text { Conformación del ingresofamiliar } \\
\text { considerando producción, } \\
\text { ingresos no agropecuarios y } \\
\text { costos totales, tanto monetarios } \\
\text { como domésticos }\end{array}$ & Cuantitativo & $\begin{array}{l}\text { smlv } \\
\text { (salario } \\
\text { mínimo legal } \\
\text { vigente) }\end{array}$ \\
\hline
\end{tabular}




\begin{tabular}{|c|c|c|c|c|}
\hline \multirow[t]{2}{*}{ Subsistema } & Variables & Concepto de la variable & Tipo de & Unidad de \\
\hline & $\begin{array}{l}\text { Relación } \\
\text { beneficio/costo }\end{array}$ & $\begin{array}{l}\text { Relación entre los Ingresos } \\
\text { brutos y los costos totales } \\
\text { (domésticos y monetarios) }\end{array}$ & Cuantitativo & $N^{2}$ \\
\hline \multirow[b]{2}{*}{ Cultural } & $\begin{array}{l}\text { Identidad } \\
\text { cultural con la } \\
\text { tierra }\end{array}$ & $\begin{array}{l}\text { Reconocimiento de la tierra como } \\
\text { un elemento culturalmente } \\
\text { sagrado o recurso explotable }\end{array}$ & Cualitativo & $\begin{array}{l}\text { Escala }+2 \text { a } \\
-2\end{array}$ \\
\hline & $\begin{array}{l}\text { Gestión de } \\
\text { conocimientos } \\
\text { técnicos y } \\
\text { prácticas } \\
\text { productivas }\end{array}$ & $\begin{array}{l}\text { Aplicación de prácticas } \\
\text { productivas soportadas en } \\
\text { criterios de innovación, } \\
\text { hibridación o tradición en la } \\
\text { gestión tecnológica del sistema } \\
\text { finca }\end{array}$ & Cualitativo & $\begin{array}{l}\text { Escala }+2 \text { a } \\
-2\end{array}$ \\
\hline \multirow{2}{*}{ Social } & $\begin{array}{l}\text { Red familiar } \\
\text { articulada al } \\
\text { sistema de } \\
\text { finca }\end{array}$ & $\begin{array}{l}\text { Participación de miembros de la } \\
\text { red familiar en el sistema finca }\end{array}$ & Cualitativo & $\begin{array}{l}\text { Escala }+2 \text { a } \\
-2\end{array}$ \\
\hline & $\begin{array}{l}\text { Residencia y } \\
\text { servicios }\end{array}$ & $\begin{array}{l}\text { Grado en que la familia se } \\
\text { beneficia del sistema finca para } \\
\text { su residencia y bienestar }\end{array}$ & Cualitativo & $\begin{array}{l}\text { Escala }+2 \text { a } \\
-2\end{array}$ \\
\hline
\end{tabular}

Fuente: el autor.

Los resultados fueron agregados obteniéndose sumatorias de variables ( $\Sigma$ var) para cada sistema de finca (basado en Fleskens et al., 2009, y Andersen et al., 2013). Para su análisis se toman como referencia los valores extremos posibles que para este caso con 10 variables son 20 y -20. Finalmente se realiza análisis estadístico de varianza para un factor (Anova) para identificar diferencias estadísticas entre los valores de las $\Sigma$ var y análisis multivariado de componentes principales para reducir la cantidad de variables que expliquen la variabilidad de los datos, usando el mismo programa SPSS versión 20. 


\section{RESULTADOS}

Entre los sistemas de finca seleccionadas (Tabla 3), las UI corresponden a formas comunitarias de tenencia de la tierra propias de las comunidades indígenas que ocupan el territorio, que corresponden a grupos descendientes de los Pijao, organizados en resguardos o áreas protegidas asignadas por el gobierno a las comunidades indígenas para su preservación como cultura. Cada resguardo determina la distribución de la tierra que incluye un área de producción comunitaria o colectiva y un área adjudicada a cada familia que no implica propiedad privada. Otra parte del área del resguardo es destinada a conservación natural. La producción comunitaria corresponde principalmente a alimentos para autoconsumo, cultivos comerciales y ganadería extensiva; emplea mano de obra comunitaria en la modalidad de "minga" que corresponde a una expresión cultural propia de los indígenas para el trabajo colectivo.

Las UF son fincas de propiedad privada o adjudicada donde generalmente la familia instala su vivienda, la mano de obra es mayoritariamente familiar, los arreglos de cultivos combinan lo tradicional con tecnología moderna.

Por su parte, las UE son manejadas por inversionistas dedicados a cultivos comerciales o ganadería bovina en tierras de su propiedad o como arrendatarios. Aplican por lo general tecnologías de agricultura moderna, acceden a créditos y su producción es casi exclusivamente para el mercado agroindustrial. 
Tabla 3. Características de las 18 fincas estudiadas en la cuenca del río Guaguarco

\begin{tabular}{|c|c|c|c|c|}
\hline Sistema de finca & Altitud (m) & $\begin{array}{l}\text { Extensión } \\
\text { (ha) }\end{array}$ & $\begin{array}{l}\text { Miembros familia/ } \\
\text { Resguardo* }\end{array}$ & Uso del suelo $0^{* *}$ \\
\hline \multicolumn{5}{|c|}{ Resguardos Indigenas } \\
\hline R. Totarco Niple & 405 & 245 & 165 & $\mathrm{PC}, \mathrm{PT}, \mathrm{PA}, \mathrm{ZC}$ \\
\hline R. Totarco Piedras & 396 & 469 & 476 & PC, PT, PM, ZC \\
\hline $\begin{array}{l}\text { R. Lomas de } \\
\text { Hilarco }\end{array}$ & 355 & 425 & 844 & $\mathrm{PM}, \mathrm{ZC}$ \\
\hline $\begin{array}{l}\text { R. Lomas de } \\
\text { Guaguarco }\end{array}$ & 337 & 147,5 & 398 & $\mathrm{PM}, \mathrm{ZC}$ \\
\hline R. Palma Alta & 352 & 240 & 383 & PM, PA, ZC \\
\hline R. El Tambo & 339 & 635 & 297 & $\mathrm{MC}, \mathrm{PM}, \mathrm{ZC}$ \\
\hline \multicolumn{5}{|l|}{ Unidades Familiares } \\
\hline El Recreo & 334 & 2,5 & 4 & PT, ZC, PC \\
\hline El lguá & 337 & 1,02 & 3 & PT, ZC \\
\hline El Cerro & 392 & 3,2 & 5 & PT, PC \\
\hline Arizona & 338 & 3 & 4 & PT,PM \\
\hline El Palmichal & 340 & 1,1 & 3 & PT, ZC \\
\hline Los Mandarinos & 341 & 1,05 & 6 & PT \\
\hline
\end{tabular}

\begin{tabular}{|l|c|c|c|c|}
\hline \multicolumn{5}{|l|}{ Unidades Empresariales } \\
\hline El Salto & 356 & 56,1 & 3 & MC, ZC, PA \\
\hline Porvenir & 337 & 12,7 & 4 & MC, ZC \\
\hline La Quinta & 332 & 45 & 4 & PM, ZC, PT \\
\hline Guamalito & 350 & 14,26 & 5 & PM, ZC \\
\hline La Esperanza & 337 & 7 & 4 & MC \\
\hline La Granja & 344 & 11,03 & 3 & PM, ZC \\
\hline
\end{tabular}

" Datos oficiales del Ministerio del Interior - Colombia. Población por res guardo a junio 30 de 2013. ** MC: Monocultivo Comercial (al godón, arroz conriego); PC: Plátano Cachaco (dedicado a la producción de hoja para tamales); PM: Pastos en Monocultivo; PT: Policultivo Tradicional; PA: Potreros Arborizados; ZC: Zona de Conservación.

Los resultados obtenidos del estudio de las 22 características analizadas de las UI, UF y UE, se muestran en la Tabla 4. 


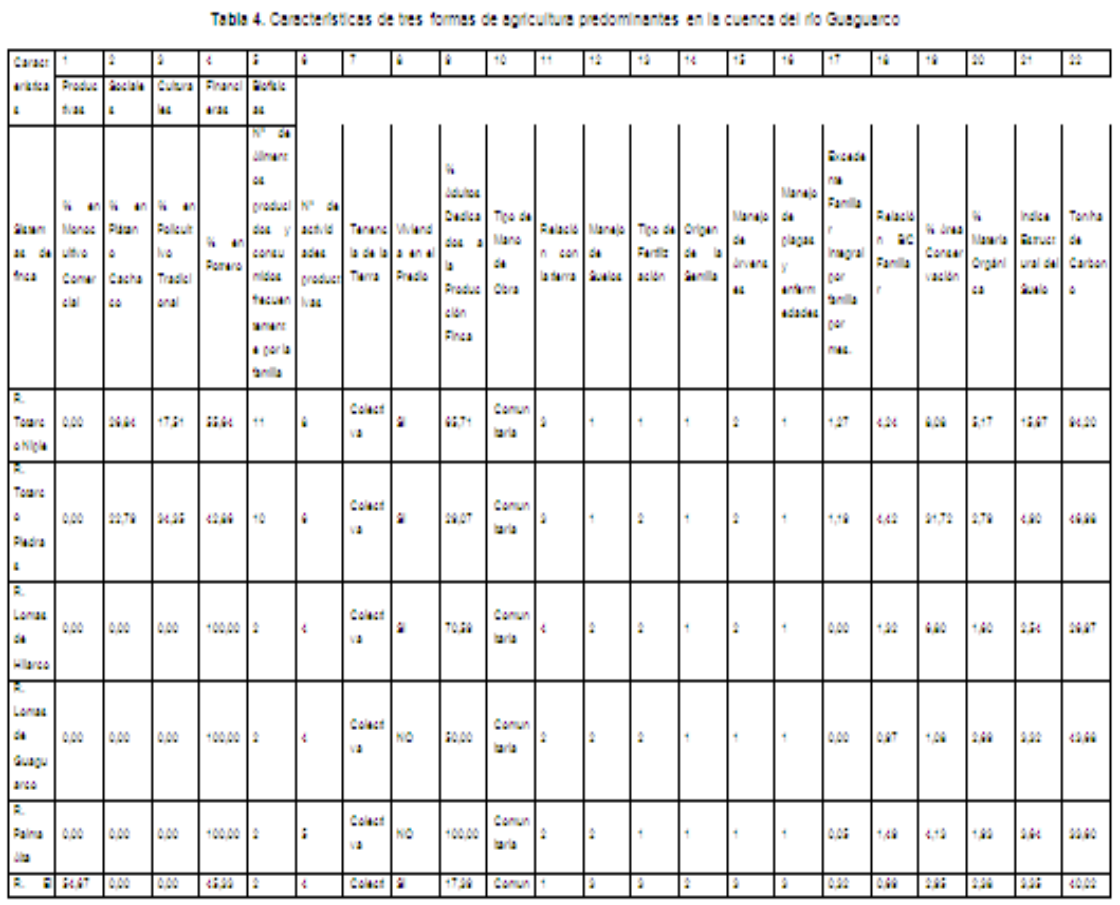

Haga clic sobre la imagen para ampliarla

En cuanto al uso del suelo se destaca que las áreas de conservación natural incluyen bosques primarios y secundarios y se ubican principalmente en la parte alta de la cuenca. Entre las 18 fincas incluidas en el estudio se presentan diversos arreglos de cultivos generalmente combinados en cada finca. En 14 de ellas existen zonas dedicadas a la conservación natural (ZC), quedan excluidas 3 unidades familiares de áreas relativamente pequeñas y una unidad empresarial cuyo encargado paga alquiler por el terreno donde produce. Tres de las 18 fincas se dedican al Monocultivo Comercial (MC), solo una (La Esperanza) tiene dedicación exclusiva al MC. Estos arreglos se presentan exclusivamente en las unidades empresariales seleccionadas. La ganadería está presente en 11 fincas, especialmente en resguardos indígenas y unidades empresariales, bien en forma de Monocultivos de Pastos (MP) o Potreros Arborizados (PA). El Policultivo Tradicional (PT) se presenta en 12 fincas incluidas todas las unidades familiares, 2 lotes comunitarios de los 6 resguardos indígenas y solo una unidad empresarial. Finalmente, las fincas productoras de plátano cachaco son 5; 2 de ellas 
corresponden a unidades familiares y 2 a resguardos indígenas de la parte alta de la cuenca.

Las áreas destinadas a conservación no varían sustancialmente entre los grupos de sistemas de finca: 9,13\% para resguardos indígenas, 7,92\% para unidades familiares y $6,33 \%$ para unidades empresariales. Sin embargo, al analizar las áreas de cultivos tradicionales, son las unidades familiares las que sobresalen con un $80,13 \%$ del área dedicada a la producción bajo sistemas agroforestales protectores del suelo y la agrobiodiversidad, seguidas de los resguardos indígenas con el $8,65 \%$, y las unidades empresariales que solo destinan en promedio el $0,56 \%$ de su área a este tipo de arreglos.

Los diversos productos que se obtienen de los sistemas de finca del territorio cuenca del río Guaguarco fueron agrupados en 10 categorías. La producción de alimento humano es predominante entre las unidades familiares (100\%), 2 resguardos indígenas y una unidad empresarial. Estos mismos sistemas de finca son los que producen y usan semillas criollas para sus propios arreglos de cultivo diversificado, evidenciándose la interrelación entre los arreglos de policultivos tradicionales y la producción de semillas criollas. La totalidad de los sistemas de finca son generadores de productos para el mercado, destacándose alimentos de pancoger, leche, ganado y hoja de plátano cachaco en la parte alta de la cuenca y ganadería, pancoger y cultivos comerciales de arroz, algodón y sorgo en la parte baja de la cuenca, como los principales renglones.

Los mayores costos de producción los evidencian las unidades empresariales debido a la alta cantidad y el alto costo de los insumos agropecuarios que emplean en sus sistemas agropecuarios. Los resguardos indígenas y unidades familiares emplean fundamentalmente insumos derivados de sus propias fincas, semillas, abonos, maderas, entre otros, reduciendo costos monetarios. Los resguardos indígenas del Tambo y Palma Alta perciben ingresos adicionales a la producción agropecuaria por alquiler parcial de sus tierras, en ambos casos para la producción de arroz bajo riego. 
La venta de fuerza de trabajo no se verifica en ninguna de las familias o resguardos que hicieron parte del estudio, lo que evidencia la preferencia de los agricultores por el trabajo en sus propios terrenos.

Los resguardos indígenas emplean en promedio 4,34 jornales/ha/año en sus lotes comunitarios especialmente en actividades vinculadas a la ganadería y manejo de potreros que constituyen la principal actividad comunitaria productiva. Esta mano de obra no es pagada, siendo la obligación de cada familia socia del resguardo aportar mano de obra regularmente según los acuerdos de la comunidad. En las unidades familiares, donde predominan policultivos tradicionales, se emplean en promedio 128,67 jornales/ha/año exclusivamente de mano de obra familiar, solo en la finca El Cerro se combina el empleo de mano de obra familiar y contratada para las labores con hoja de plátano cachaco. En las unidades empresariales se utilizan 60,23 jornales/ha/año pagados en su totalidad. Especialmente en este último grupo se nota una gran dispersión de los datos resaltándose dos grupos, el primero conformado por las fincas que emplean entre 5,53 y 20,76 jornales dedicadas principalmente a la ganadería y el segundo las que emplean entre 47,5 y 149,6 jornales/ha/año que corresponden a fincas donde cultivan monocultivos comerciales de arroz y algodón principalmente.

En general, los contenidos de materia orgánica encontrados en los suelos son bajos, entre $0,74 \%$ y $5,17 \%$ si se tiene en cuenta su origen volcánico. Los promedios por tipo de sistema productivo muestran el mayor contenido porcentual de materia orgánica en las fincas de los resguardos indígenas $(2,76 \%)$ seguida de las fincas familiares $(2,37 \%)$ y el menor valor promedio es para las unidades empresariales $(1,48 \%)$.

El análisis de conglomerados conformó tres grupos de sistemas de finca, diferenciados por un total de 19 de las 22 características que resultaron determinantes para la agrupación. Siete características tienen una alta significancia en la conformación de los grupos; entre estas se cuentan 5 del subsistema productivo (número de alimentos para autoconsumo, \% del área en potreros, \% del área en policultivos tradicionales, \% del área en monocultivo comercial y número de actividades productivas) y 2 características del subsistema cultural (tipo de conocimiento en el manejo de suelos y tipo de conocimientos en la fertilización). 
Esto muestra que las características que marcan las mayores diferencias entre los conglomerados están relacionadas principalmente a la configuración de la producción y el tipo de conocimientos empleados en la gestión de la producción.

Aunque los sistemas de finca fueron seleccionados inicialmente por distintivos como tenencia de la tierra, arreglos productivos, mano de obra, destino de la producción y tecnologías empleadas, distinguiendo unidades familiares cercanas a la idea de condición campesina (Toledo, 1993; Ploeg, 2008; Forero, 2010); predios dedicados al capitalismo rural definidos como unidades empresariales (Ploeg, 2008; Forero, 2010) y predios de comunidades indígenas, el análisis de conglomerado realizado permite un reagrupamiento más riguroso respecto a la clasificación original en UI, UF y UE; en esta investigación se reagrupan sistemas de agricultura tradicional (conglomerado 1) con 8 sistemas de finca, 2 UI y 6 UF dedicadas a agricultura tradicional; sistemas ganaderos (conglomerado 2) conformado con un total de 6 fincas: 2 UI y 3 UE que dedican su área comunitaria o privada a ganadería y sistemas de monocultivo semestral de arroz, algodón y sorgo (conglomerado 3 ) que consta de 4 fincas, entre ellas una UI y 3 UE.

De esta manera, se puede comprobar cómo el territorio en la cuenca del río Guaguarco se muestra estructurado por formas productivas híbridas, como mosaicos sociales y físicos complejos (Forero, 2010) o antropobiomas (Ellis \& Ramankutty, 2008; Feijoo, Quintero \& Forero, 2010) que combinan características de la producción familiar campesina con formas más cercanas al mercado y a la generación de renta.

Los resultados de evaluación de multifuncionalidad para los sistemas de agricultura tradicional muestran un comportamiento positivo generalizado de las variables. Totarco Niple es el único sistema que muestra un comportamiento positivo en la totalidad de las variables, las demás tienen entre una y tres variables en negativo, es decir en el área de afectación sobre la funcionalidad del sistema (Figura 2). 

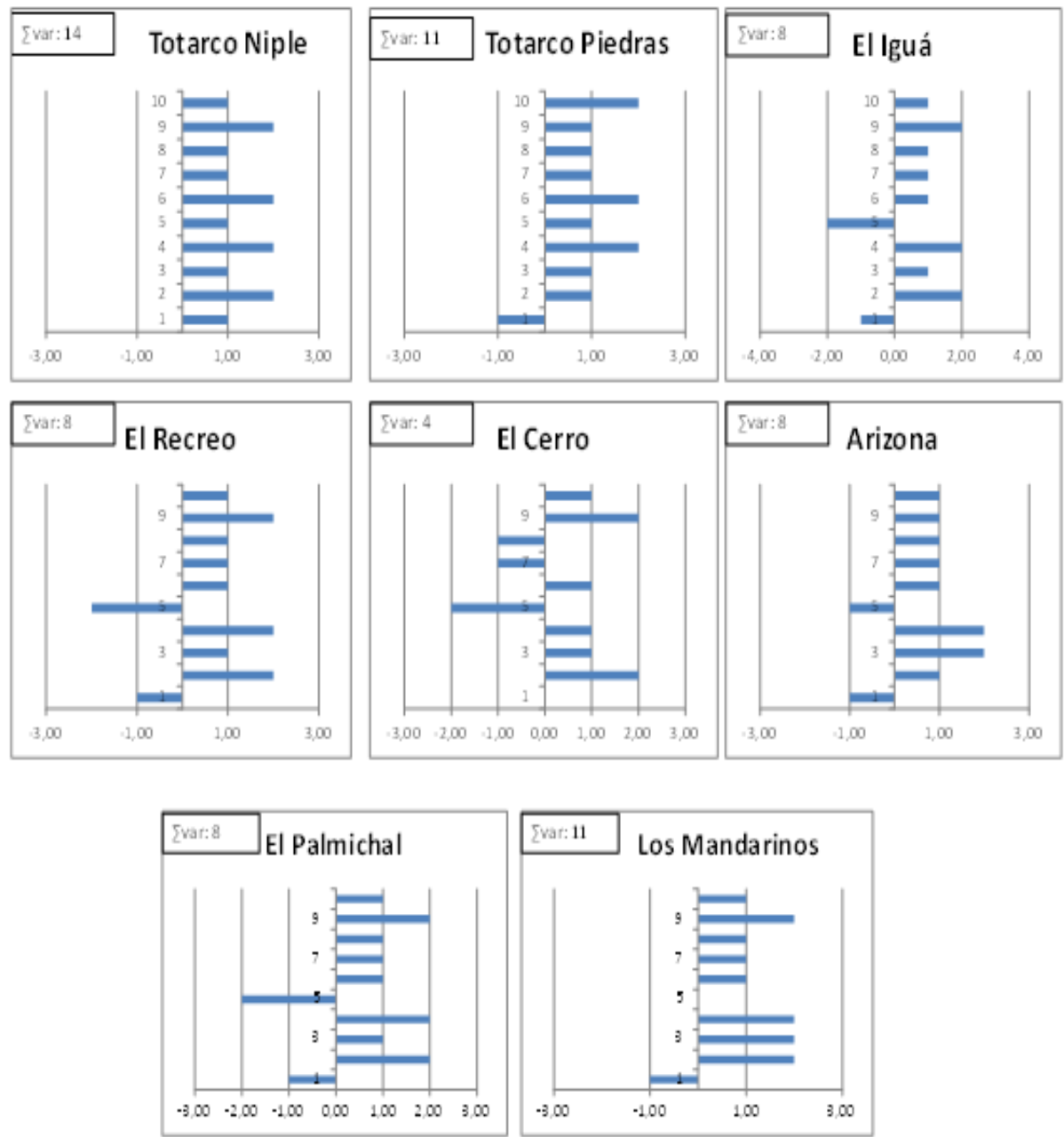

¿var: Sumatoria de variables. 1. Contenidos de materia orgánica. 2. Cobertura de conservación. 3. Diversificación productiva. 4. Abastecimiento alimentario. 5. Excedente familiarintegral mensual. 6. Relación beneficio/costo del sistema agropecuario. 7. Representación cultural de la tierra. 8. Gestión de conocimientos técnicos y prácticas productivas. 9. Red familiar articulada al sistema finca. 10. Residencia y servicios.

\section{Fuente: el autor.}

Figura 2. Evaluación de funciones múltiples de la agricultura en sistemas de agricultura tradicional, cuenca del río Guaguarco.

En los sistemas de agricultura tradicional, todos sus componentes o subsistemas, aportan a su funcionalidad. Los subsistemas productivo y social, en su orden, son las que más la determinan; el subsistema cultural muestra una contribución moderada a la funcionalidad de los sistemas de agricultura tradicional; mientras 
que las variables de los subsistemas biofísico y financiero son las que menos la están determinando.

Aunque los agricultores tradicionales no reducen la tierra exclusivamente a la única función financiera (Pérez-Vitoria, 2010), en los casos estudiados combinan valores de uso con mercancías para la supervivencia de la unidad doméstica campesina (Toledo, 1993); usan, por ejemplo, herbicidas para el control de malezas en cultivos de hoja de plátano cachaco para ahorrar tiempo de los brazos fuertes y liberarlo para otros trabajos dentro o fuera del sistema de finca (Zúñiga, FeijooMartínez \& Quintero, 2009). También, los agricultores tradicionales recurren a la pluriactividad (Ploeg, 2008; Schneider, 2009) para suplementar sus ingresos mediante la venta de productos de extracción o actividades artesanales.

Por su parte, los sistemas ganaderos muestran tendencias negativas en la mayoría de las variables, desde sistemas de finca con 3 variables en el campo negativo hasta sistemas con 9 variables. La variable relación beneficio/costo se muestra en el área positiva en 5 de los 6 casos, mientras que la variable red familiar integrada al sistema de finca se muestra positiva en 3 de las 6 fincas (Figura 3). 

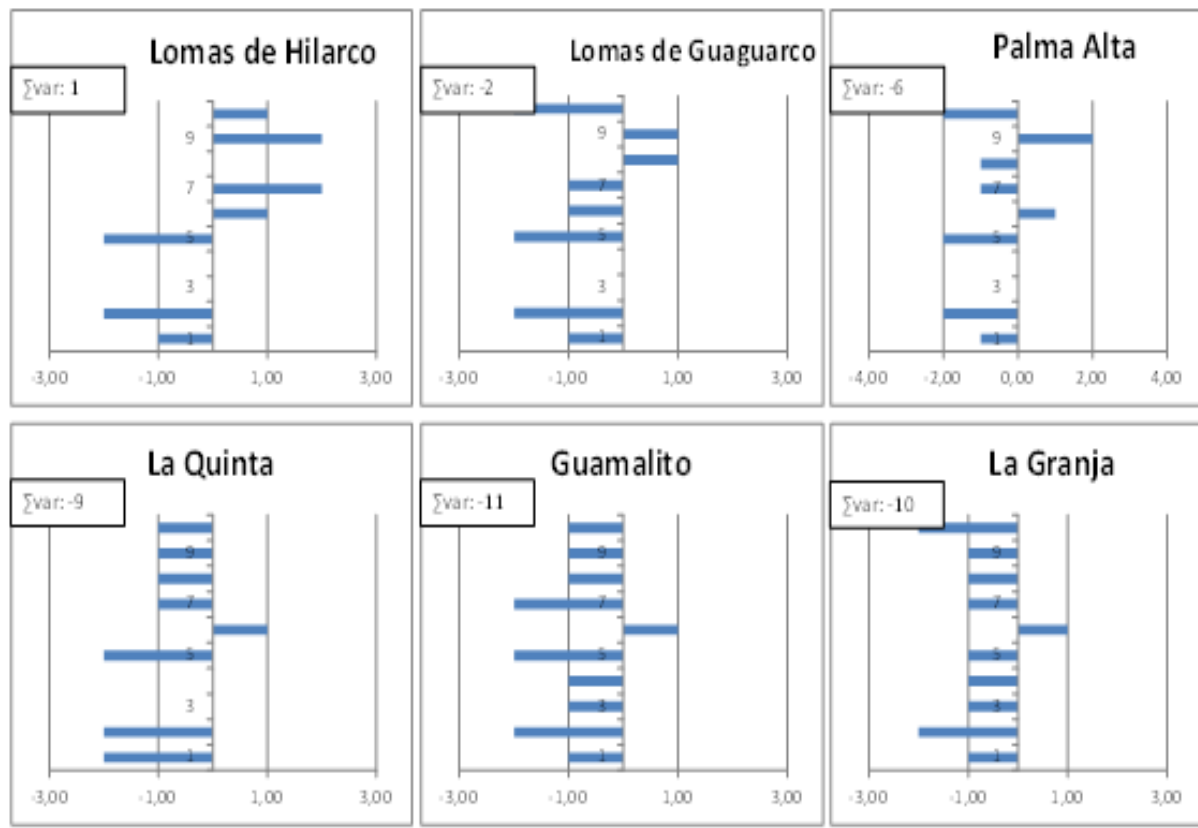

Evar: Sumatoria de variables. 1. Contenidos de materia orgánica. 2. Cobertura de conservación. 3. Diversificación productiva. 4. Abastecimiento alimentario. 5. Excedente familiarintegral mensual. 6. Relación beneficio/costo del sistema agropecuario. 7. Representación cultural de la tierra. 8. Gestión de conocimientos técnicos y prácticas productivas. 9. Red familiar articulada al sistema finca. 10. Residencia y servicios.

Fuente: el autor.

Figura 3. Evaluación de funciones múltiples de la agricultura en sistemas ganaderos, cuenca del río Guaguarco

Los sistemas ganaderos, practicados por agricultores inversionistas o comunidades indígenas en la zona, se basan en una lógica de aprovechamiento natural del terreno, con baja inversión y poco laboreo. Después de los fracasos repetidos de la agricultura de monocultivos comerciales de los años noventa, muchos agricultores de la región decidieron potrerizar sus terrenos y criar ganado, con lo cual disminuyen el riesgo de pérdida de la inversión. Sobre esta misma lógica, muchas comunidades indígenas del territorio destinaron también sus áreas colectivas a esta actividad, práctica muy contraria a la que predomina en las áreas adjudicadas a la familia, que destinan a la producción de alimentos. 
Los sistemas de monocultivos semestrales muestran un comportamiento generalizado de las variables en el área negativa, afectando entre leve y fuertemente la funcionalidad de los sistemas de finca (Figura 4). Las variables financieras muestran un comportamiento positivo en 3 de los 4 sistemas del conglomerado. El Tambo muestra un comportamiento diferente a las tres fincas restantes del grupo, evidenciando que son las variables del subsistema social las que se muestran en el campo positivo (red articulada al sistema productivo y residencia y servicios), lo que denota la fuerza que tienen estas variables en la funcionalidad de dicho sistema de finca de tipo comunitario. Todos los sistemas de finca de este conglomerado muestran $\sum$ var negativas.

Los subsistemas biofísico, productivo y cultural son los que más determinan el comportamiento negativo de la multifuncionalidad en estos sistemas de finca; el subsistema social la afecta en menor grado; las dos variables del subsistema financiero son las que determinan su funcionalidad. Los monocultivos comerciales se construyen sobre una racionalidad diferente a la de los agricultores tradicionales, más inmediatista, menos preocupada por el bienestar social o ambiental y más centrada en la economía del negocio agropecuario con rendimientos en el corto plazo. Con estas consideraciones, los agricultores acceden a las tecnologías de producto con costos económicos altos que inducen a una mayor producción por la manera como estas innovaciones aceleran los ciclos productivos, pero comprometen la rentabilidad económica y la estabilidad ambiental a largo plazo. 

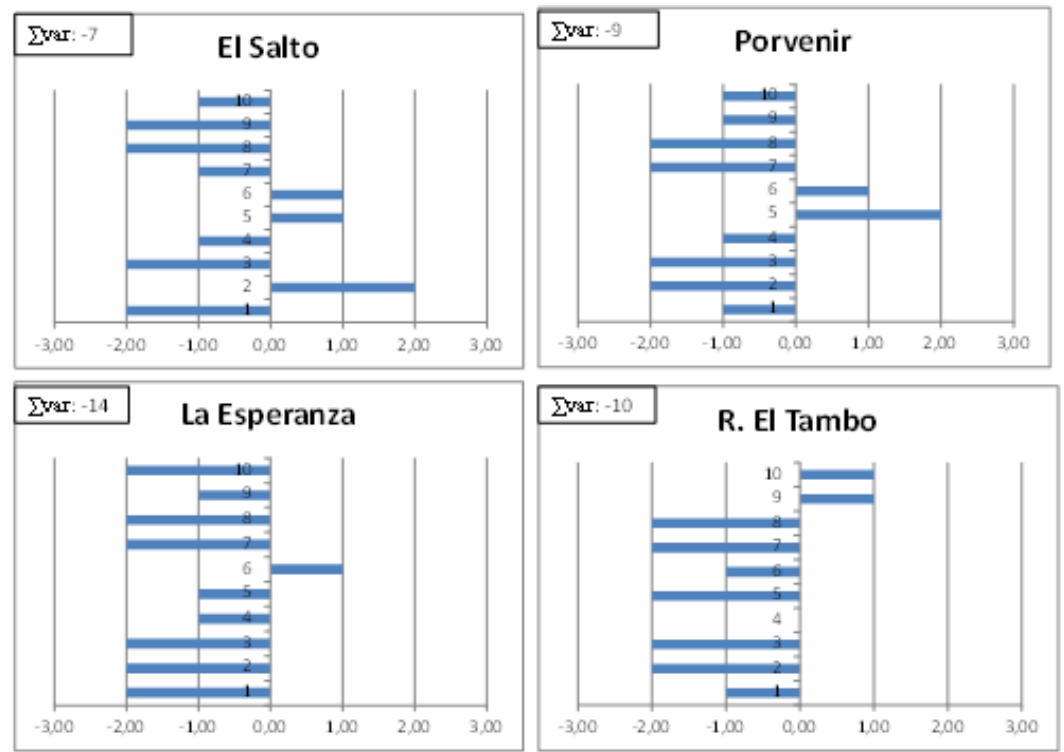

¿var: Sumatoria de variables. 1. Contenidos de materia orgánica. 2. Cobertura de conservación. 3. Diversificación productiva. 4. Abastecimiento alimentario. 5. Excedente familiarintegral mensual. 6. Relación beneficio/costo del sistema agropecuario. 7. Representación cultural de la tierra. 8. Gestión de conocimientos técnicos y prácticas productivas. 9 . Red familiar articulada al sistema finca. 10. Residencia y servicios.

$$
\text { Fuente: el autor. }
$$

Figura 4. Evaluación de funciones múltiples de la agricultura en sistemas de monocultivos semestrales, cuenca del río Guaguarco.

Siguiendo los criterios de la escala de análisis de multifuncionalidad propuesta por Lovell et al. (2010) es posible interpretar que los valores negativos de $\sum$ var en varios sistemas, no contribuyen sino que afectan la funcionalidad de los sistemas por lo que se pueden interpretar también como no funcionales, casos concretos de los conglomerados de sistemas ganaderos $y$, en mayor grado, monocultivos semestrales.

En el análisis estadístico multivariado por componentes principales, por su parte, las 10 variables de multifuncionalidad que integran el estudio son resumidas en dos componentes o nuevas variables que explican el $76,16 \%$ de la varianza total explicada por el comportamiento de los datos (Tabla 5), es decir que estos dos componentes describen suficientemente el conjunto de las 10 variables sin perder información importante. Las cargas factoriales más significativas de la matriz 
muestran dos componentes, el primero de los cuales explica fuertemente la variabilidad de las variables de los subsistemas biofísico, productivo, social y cultural; mientras que el segundo componente explica la variabilidad de las variables del subsistema financiero.

Tabla 5. Análisis de componentes principales para 10 variables de funciones múltiples de la agricultura, cuenca del río Guaguarco

\begin{tabular}{|l|c|c|c|}
\hline \multirow{2}{*}{ Variables de FMA } & \multicolumn{2}{|c|}{ Componentes } & \multirow{2}{*}{ Comunalidades } \\
\cline { 2 - 3 } & $\mathbf{1}$ & $\mathbf{2}$ & 0,923 \\
\hline $\begin{array}{l}\text { Abastecimiento } \\
\text { alimentario }\end{array}$ & 0,960 & $-0,033$ & 0,905 \\
\hline $\begin{array}{l}\text { Cobertura } \\
\text { conservación }\end{array}$ & 0,944 & 0,121 & 0,859 \\
\hline $\begin{array}{l}\text { Diversificación } \\
\text { productiva }\end{array}$ & 0,922 & $-0,098$ & 0,824 \\
\hline $\begin{array}{l}\text { Red familiar articulada } \\
\text { conocimientos }\end{array}$ & 0,822 & $-0,385$ & 0,816 \\
\hline $\begin{array}{l}\text { Excedente familiar } \\
\text { integral mensual }\end{array}$ & $-0,042$ & 0,889 & 0,791 \\
\hline $\begin{array}{l}\text { Representación cultural } \\
\text { de la tierra }\end{array}$ & 0,882 & 0,063 & 0,781 \\
\hline Relación b/c & 0,392 & 0,768 & 0,744 \\
\hline Residencia y servicios & 0,794 & 0,068 & 0,636 \\
\hline Materia orgánica & 0,571 & 0,101 & 0,336 \\
\hline Varianza & 60,285 & 15,873 & Acum.: 76,16\% \\
\hline
\end{tabular}

Fuente: el autor.

Los promedios de $\sum$ var destacan los sistemas agrícolas tradicionales como multifuncionales $(8,63)$, seguidos de los sistemas ganaderos $(-6,00)$ y por último el conglomerado de sistemas de monocultivo semestral $(-11,00)$; mientras que el análisis estadístico de varianza para un factor reporta diferencias entre los sistemas de agricultura tradicional con los ganaderos y los de monocultivos 
semestrales, pero no se reporta diferencia significativa para la comparación entre las $\sum$ var para los sistemas ganaderos y sistemas de monocultivos semestrales (Tabla 6).

Tabla 6. Anova de un factor para comparar multifuncionalidad en 3 sistemas de finca de la cuenca del río Guaguarco

\begin{tabular}{|l|l|c|c|c|}
\hline \multicolumn{2}{|l|}{ Sistema finca } & $\begin{array}{c}\text { Diferencia de } \\
\text { medias }\end{array}$ & Error típico & Sig. \\
\hline $\begin{array}{l}\text { Agricultura } \\
\text { tradicional }\end{array}$ & $\begin{array}{l}\text { Sistemas } \\
\text { ganaderos }\end{array}$ & $14,62500^{\circ}$ & 1,96152 & 0,000 \\
\cline { 2 - 5 } & $\begin{array}{l}\text { Monocultivos } \\
\text { semestrales }\end{array}$ & $19,62500^{\circ}$ & 2,22416 & 0,000 \\
\hline $\begin{array}{l}\text { Sistemas } \\
\text { ganaderos }\end{array}$ & $\begin{array}{l}\text { Agricultura } \\
\text { tradicional }\end{array}$ & $-14,62500^{-}$ & 1,96152 & 0,000 \\
\cline { 2 - 5 } & $\begin{array}{l}\text { Monocultivos } \\
\text { semestrales }\end{array}$ & 5,00000 & 2,34447 & 0,117 \\
\hline $\begin{array}{l}\text { Monocultivos } \\
\text { semestrales }\end{array}$ & $\begin{array}{l}\text { Agricultura } \\
\text { tradicional }\end{array}$ & $-19,62500^{-}$ & 2,22416 & 0,000 \\
\cline { 2 - 5 } & $\begin{array}{l}\text { Sistemas } \\
\text { ganaderos }\end{array}$ & $-5,00000$ & 2,34447 & 0,117 \\
\hline
\end{tabular}

- La diferencia de medias es significativa al nivel 0,05 .

Fuente: el autor usando el programa SPSS (vers. 20).

Los resultados de este estudio indican que la multifuncionalidad se desarrolla en mejores condiciones bajo esquemas de agricultura tradicional, circunstancia que coincide con lo demostrado por Ayala-Ortiz \& García-Barrios (2009) en una evaluación comparativa de multifuncionalidad para comunidades indígenas y no indígenas en México; situación que los autores explican por las costumbres, prácticas y normas que constituyen la base de la multifuncionalidad sociocultural y del capital social de estas comunidades. 
Los agricultores empresariales que plantan monocultivos semestrales tienden a una especialización productiva con alto nivel de explotación de sus recursos; esta simplificación a que obligó la tendencia productivista, hizo que estos sistemas de finca perdieran su multifuncionalidad, reduciéndolos a la mera función financiera (Naredo, 2010). Por el contrario, los agricultores tradicionales tienden a una producción diversificada que optimiza el uso de sus recursos y permite la regulación natural de sus ecosistemas mediante el reciclaje de materias, energía, agua y residuos (Toledo, 1993).

Varios autores interpretan los resultados de la evaluación de multifuncionalidad centrados en cuantificar la cantidad de funciones de cada sistema de finca; sin embargo, en un análisis que supere este enfoque de contabilidad es posible interpretar la funcionalidad de los sistemas en los polos de multifuncionalidad cuando estas aportan a la estabilidad total el sistema de finca garantizando su continuidad y generando exterioridades positivas a la sociedad y el ambiente, y el de disfuncionalidad si los efectos generados desestabilizan el sistema haciéndolo vulnerable y sobrepasan el ámbito del sistema de finca impactando adversamente a la sociedad.

La disfuncionalidad relativa de los sistemas ganaderos y de monocultivos comerciales, se genera por la menor relación entre los componentes del sistema, sin desplegar funciones más allá de la financiera. Estas formas de agricultura generan un altísima pérdida de la biodiversidad, y esta constituye una de las más serias amenazas para la estabilidad de la agricultura a futuro (IASSTAD, 2009).

Las investigaciones desarrolladas sobre multifuncionalidad en la agricultura (Gómez-Sal \& González, 2007; Fleskens et al., 2009; Lovell et al., 2010; Andersen et al., 2013) se dedican a valorar las funciones de la agricultura y a hacer visibles las exterioridades positivas buscando regulaciones desde el mercado, instrumentalizando así el concepto. En América Latina, lejos de una agricultura regulada por subsidios, un sentido útil de la multifuncionalidad puede ser construido más allá de la cuantificación de las funciones para abordar un análisis sobre la manera como los agricultores configuran los sistemas de finca para hacerlos altamente funcionales de acuerdo con sus posibilidades y 
expectativas, así como del contexto biofísico y cultural que ocupan generando una interacción compleja entre los factores que la determinan.

El enfoque de la multifuncionalidad centrado en los actores sociales (Renting et al., 2009), tomado para esta investigación, analizó aspectos de las agriculturas en la cuenca del río Guaguarco a partir de las ciencias sociales y la agronomía, permitiendo una mirada integrada de lo que ocurre en términos productivos y sociales. Desde este enfoque, es posible abordar el estudio de las distintas formas de agricultura en un territorio para comprender la racionalidad de los agricultores respecto al manejo de sus sistemas de finca con el despliegue de funciones múltiples que se complementan entre sí para regular el funcionamiento total del sistema que les da sustento y bienestar.

El enfoque de multifuncionalidad de la agricultura para las condiciones de América Latina debe entonces emplearse como un enfoque analítico para comprender la racionalidad y capacidad adaptativa de los agricultores en la gestión de sus fincas y los territorios para hacerlos multifuncionales de acuerdo a sus posibilidades y expectativas generando sostenibilidad.

\section{CONCLUSIONES}

La caracterización multifuncional de la estructura y funcionamiento de los sistemas de finca permitió identificar las formas de agricultura que prevalecen en la cuenca del río Guaguarco, sus características, mezclas, adaptaciones e innovaciones generadas por los propios agricultores; pudiéndose establecer que no existe una masa homogénea de sistemas sino un mosaico de formas productivas híbridas a lo largo de toda la cuenca, en las que cada familia agricultora aplica estrategias productivas de acuerdo a su cultura y sus necesidades particulares, tamaño del predio, racionalidad ecológica, económica y productiva, que fueron agrupadas 
como sistemas de agricultura tradicional, sistemas de producción ganadera y sistemas de monocultivos semestrales.

La evaluación de multifuncionalidad de estas formas de agricultura demuestra que existen diversos grados de funcionalidad para cada una; mientras que los sistemas de agricultura tradicional se muestran multifuncionales (promedio de variables de 8,63 ), los sistemas ganaderos (promedio de variables de $-6,0$ ) y de monocultivos semestrales (promedio de variables de -11,0) se muestran poco funcionales generando externalidades negativas que comprometen su estabilidad en el tiempo.

La multifuncionalidad de la agricultura tradicional en la cuenca tienen que ver con la diversificación de la producción y el otorgamiento de valores de consumo, protección y bienestar; valores que son minimizados por los agricultores de los sistemas ganaderos y de monocultivos semestrales.

La exclusiva y convencional evaluación de rentabilidad financiera de los sistemas de finca deja de lado la ponderación de muchas funciones que cumple la agricultura y que la hacen viable en su conjunto. La evaluación de multifuncionalidad enfatiza la capacidad de analizar los sistemas de finca más allá de sus funciones de producción agrícola y su valor económico convencional, permitiendo la incorporación en los análisis de medidas de conservación de los recursos naturales, además de medidas culturales y sociales que facilitan una mejor comprensión y abordaje de diferentes aspectos del desarrollo sustentable de la agricultura y las áreas rurales.

La capacidad de los agricultores de otorgar a sus sistemas de finca múltiples funciones, ajustando permanentemente su estructura, constituye una estrategia para enfrentar las adversidades externas que ponen en riesgo la continuidad de las operaciones y la capacidad de los sistemas de producir bienes y servicios para el bienestar social. Las decisiones sobre el funcionamiento de los sistemas no están basadas exclusivamente en aspectos técnicos, se regulan también por consideraciones de tipo cultural, ambiental y social al mismo tiempo. 
Más allá de las valoraciones de funciones, el concepto de multifuncionalidad permite analizar la manera como los agricultores configuran los sistemas de finca para hacerlos funcionales de acuerdo a sus posibilidades y expectativas.

\section{AGRADECIMIENTOS}

Especial agradecimiento a los doctores Álvaro Rivas, Heimar Quintero, María Clara van der Hammen, Peter Gerritsen, León Darío Vélez y Alejandro Aguilar por sus contribuciones en la realización de esta investigación.

\section{POTENCIAL CONFLICTO DE INTERESES}

El autor manifiesta que no existe conflicto de interés alguno respecto al presente artículo.

\section{FUENTES DE FINANCIACIÓN}

El proyecto de investigación no contó con financiación institucional alguna. 


\section{REFERENCIAS}

- Aguirre, E. (1998). Representaciones sociales. Bogotá: UNAD, Facultad de Ciencias Sociales y Humanas.

- $\quad$ Andersen, P. S. Vejre, H. Dalgaard, T. \& Brandt, J. (2013). An Indicator-based method for quantifying farm multifunctionality. Ecological Indicators Magazine, 25, 166-179.

- Ayala-Ortiz, D. A., \& García-Barrios, R. (2009). Contribuciones metodológicas para valorar la multifuncionalidad de la agricultura campesina en la Meseta Purépecha. Economía, Sociedad y Territorio, 9(31), 759-801.

- Baudel, M. de N. (2009). O agricultor familiar no Brasil: um ator social da construção do futuro. En Agricultura familiar camponesa na construção do futuro (pp. 33-46). Rio de Janeiro: AS-PTA.

- Bjørkhaug, H., \& Richards, C. (2008). Multifunctional agriculture in policy and practice? A comparative analysis of Norway and Australia. Journal of Rural Studies, 24, 98-111.

- Ellis, E. C., \& Ramankutty, N. (2008). Putting people in the map: anthropogenic biomes of the World. Frontiers in Ecology and the Environment, 6(8), 439-447.

- Feijoo, A., Quintero, H., \& Forero, C. (2010). Dinámica espacial en antropobiomas de los Llanos Orientales, Colombia. Acta biológica Venezuelica, 30(1-2), 11-21. 
- Fleskens, L. Duarte, F., \& Eicher, I. (2009). A conceptual framework for the assessment of multiple functions of agro-ecosystems: A case study of Trás-osMontes olive groves.Journal of Rural Studies, 25, 141-155.

- Forero, J. (2002). La economía campesina colombiana 1990-2001. Bogotá: ILSA.

- Forero, J. (2010). El campesino colombiano: entre el protagonismo económico y el desconocimiento de la sociedad. Bogotá: Facultad de Estudios Ambientales y Rurales, Pontifica Universidad Javeriana.

- Gerritsen, P., Rosales, A., Moreno, L. M., \& Martínez, R. (2006). Sistemas productivos y sustentabilidad rural en la costa sur de Jalisco en el occidente de México. $11^{\circ}$ Encuentro Nacional sobre Desarrollo Regional en México. Mérida, Yucatán.

- Gómez, M. A., Deslauriers, J. P., \& Alzate, M. V. (2010). Cómo hacer tesis de maestría y doctorado. Bogotá: Ecoe Ediciones.

- Gómez-Sal, A., \& González, A. (2007). A comprehensive assessment of multifunctional agricultural land-use systems in Spain using a multi-dimensional evaluative model.Agriculture, Ecosystems and Environment, 120, 82-91.

- Hart, R. (1991). Componentes, subsistemas y propiedades del sistema finca como base para un método de clasificación. En G. Escobar, J. Berdagué (Eds.), Tipificación de sistemas de producción agrícola (pp. 45-62). Santiago de Chile: RIMISP.

- Hernández, A., Vargas, D., Borges, Y., Ríos, H., Morales, M., \& FunesMonzote, F. R. (2011). Reservas de carbono orgánico en suelos ferralíticos rojos. En H. Ríos, D. Vargas, F. R. Funes-Monzote (Eds.), Innovación agroecológica, adaptación y mitigación del cambio climático (pp. 45-55). La Habana: Instituto Nacional de Ciencias Agrícolas. 
- Holmes, J. (2006). Impulses towards a multifunctional transition in rural Australia: gaps in the research agenda. Journal of Rural Studies, 22, 142160.

- $\quad$ IAASTD -International Assessment of Agricultural Knowledge, Science and technology for Development-. (2009). Agriculture at a crossroads.Global report. Washington: UNDP, FAO, UNEP, UNESCO.

- Jordan, N., \& Warner, K. D. (2010). Enhancing the multifunctionality of US agriculture.BioScience, 60, 60-66.

- Labarthe, P. (2009). Extension services and multifunctional agriculture. Lessons learnt from the French and Dutch contexts and approaches. Journal of Environmental Management, 30, 1-10.

- Licona, I. (2012). Transformación del sistema agrario y su multifuncionalidad en dos comunidades indígenas: Cuzalapa y Ayotitlán, Jalisco. (Tesis de maestría). Universidad Iberoamericana, Puebla, México.

- Lovell, S. T., DeSantis, S., Nathan, C. A., Breton, M., Méndez, E., Kominami, H. C.,... Morris, W. (2010). Integrating agroecology and landscape multifunctionality in Vermont: an evolving framework to evaluate the design of agroecosystems. Agricultural Systems, 103, 327-341.

- Moyano, E. (2008). Multifuncionalidad, territorio y desarrollo de las áreas rurales.Ambienta, $\quad 81, \quad 6-20 . \quad$ Recuperado de http://www.mma.es/secciones/biblioteca publicacion/ publicaciones/revista ambienta/n81/pdf/06-19 Espania CSIC.pdf

- Murillo, B. E. (2010). Disponibilidad de recursos y tipos de sistemas de cultivo de café y plátano en la cuenca del río La Vieja, Colombia. (Tesis de maestría). Facultad de Ciencias Ambientales, Universidad Tecnológica de Pereira, Pereira. 
- Naredo, J. M. (2010). Raíces económicas del deterioro ecológico y social. Más allá de los dogmas. Madrid: Siglo XXI.

- OECD. (2001). Multifunctionality: towards an analytical framework. Paris: Organization for Economic Cooperation and Development.

- Pérez-Vitoria, S. (2009). El retorno de los campesinos. Una oportunidad para nuestra supervivencia. Barcelona: Icaria.

- $\quad$ Perry, S., Barberi, F., \& Garay, L. J. (2013). Propuestas de política pública para el desarrollo de la economía campesina en Colombia. En L. J. Garay, R. Bailey, J. Forero, F. Barberi, C. Ramírez, M. D. Suárez,... S. Perry. Reflexiones sobre la ruralidad $y$ el territorio en Colombia. Problemáticas y retos actuales (pp. 367-407). Bogotá: OXFAM.

- Pieri, C. (1995). Long-term management experiments in semi-arid Francophone Africa. En R. Lal, B. A. Stewart (Eds.), Soil management. Experimental basis for sustainability and environmental quality (pp. 225266). EEUU: Lewis Publishers.

- Ploeg, J. D. van der. (2008). Camponeses e impérios alimentares. Lutas por autonomia e sustentabilidades na era da globalização. Porto Alegre: Universidade Federal do Rio Grande do Sul.

- Renting, H., Rossing, W. A. H., Groot, J. C. J., Ploeg, J. D. van der, Laurent, C., Perraud, D.,... Van Ittersum, M.K. (2009). Exploring multifunctional agriculture.A review of conceptual approaches and prospects for an integrative transitional framework. Journal of Environmental Management, XXX, 1-12.

- Rivas, A., \& Quintero, H. (2014). Reappraising the multiple functions of traditional agriculture within the context of building development investigative skills. Agronomía Colombiana, 32(1), 130-137. 
- Samper, M., \& Torrens, J. (2015). Políticas públicas para el desarrollo de los Territorios rurales. En Un recorrido por líneas locales. Aportes para políticas públicas en el sector rural de Centroamérica, el Caribe y la Región Andina (pp. 7-23). Recuperado de http://cdr.or.cr/libro/completo.pdf

- Schneider, S. (2009). A pluriactividade na agricultura familiar. Porto Alegre, Brasil: UFRGS.

- $\quad$ Steiner, G., \& Posch, A. (2006). Higher education for sustainability by means of transdisciplinary case studies: an innovative approach for solving complex, real-world problems. Journal of Cleaner Production, 14(9-11), 877-890.

- Toledo, V. M. (1993). La racionalidad ecológica de la producción campesina. En E. Sevilla, M. González (Eds.), Ecología, campesinado e historia (pp. 197-218). Madrid: La Piqueta.

- Toledo, V. M., Alarcón-Cháires, P., \& Barón, L. (2009). Revisualizar lo rural desde una perspectiva multidisciplinaria. Polis, revista de la Universidad Bolivariana, 8(23), 328-345.

- Toledo, V. M., \& Barrera-Bassols, N. (2008). La memoria bio-cultural. La importancia ecológica de las sabidurías tradicionales. Barcelona: Icaria.

- Wilson, G. (2009). The spatiality of multifunctional agriculture: a human geography perspective. Geoforum, 40, 269-280.

- Zúñiga, M. C., Feijoo-Martínez, A., \& Quintero, H. (2009). Estructuras familiares en agroecosistemas en la cuenca del río La Vieja, Colombia. En Valoración de la biodiversidad en la ecorregión del Eje Cafetero. Pereira: CIEBREG. 
1. Máster en Agroecología y Desarrollo Rural Sostenible. Candidato a Doctor en Agroecología, Universidad Nacional de Colombia. Bogotá, Colombia. aacevedoo@unal.edu.co. ORCID: 0000-0003-2132-4891

2. Se emplea el término "Sistema de Finca" para dar una idea multidimensional de lo que representa la agricultura para las familias y comunidades más allá de su mera función productiva. El término es tomado de Hart (1991) para quien "Las fincas son sistemas con diferentes tipos de recursos, procesos y componentes que los agricultores, individual o colectivamente, combinan para formar subsistemas" (p. 48).

Para citar este artículo: Acevedo-Osorio, Á. (2016). Monofuncionalidad, multifuncionalidad e hibridación de funciones de las agriculturas en la cuenca del río Guaguarco, sur del Tolima.Revista Luna Azul, 43, 251-285. Recuperado de http://200.21.104.25/lunazul/index.php?option=com_content\&view=article\&id=201

Esta obra está bajo una Licencia de Creative Commons Reconocimiento CC BY

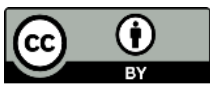

